\title{
Iterative Synthesis of 1,3-Polyboronic Esters with High Stereocontrol: Applications to Bahamaolide A and Polyfunctionalised Hydrocarbons
}

\author{
Sheenagh G. Aiken ${ }^{\dagger}$ Joseph M. Bateman ${ }^{\dagger}$, Hsuan-Hung Liao ${ }^{\dagger}$, Alexander Fawcett, Teerawut \\ Bootwicha, Paolo Vincetti, Eddie L. Myers, Adam Noble, Varinder K. Aggarwal*
}

†These authors contributed equally to this work.

School of Chemistry, University of Bristol, Cantock’s Close, Bristol BS8 1TS, UK.

\begin{abstract}
Polyketide natural products often contain common repeat motifs, e.g., propionate, acetate and deoxypropionate, and so can be synthesized by using iterative processes. We report a highly efficient iterative strategy for the synthesis of polyacetates based on boronic ester homologation that does not require functional group manipulation between iterations. This process involves sequential asymmetric diboration of a terminal alkene, forming a 1,2-bis(boronic ester), followed by regio- and stereoselective homologation of the primary boronic ester with a butenyl metallated carbenoid to generate a 1,3bis(boronic ester). Each transformation independently controls stereochemical configuration, making the process highly versatile, and the sequence can be iterated prior to stereospecific oxidation of the 1,3-poly(boronic ester) to reveal the 1,3-polyol. The methodology was applied to a 14-step synthesis of the oxopolyene macrolide bahamaolide $\mathrm{A}$, and the versatility of the 1,3-polyboronic esters was demonstrated in various stereospecific transformations, leading to poly-alkenes, -alkynes, -ketones, and -aromatics with full stereocontrol.
\end{abstract}

\section{MAIN TEXT}

Since erythromycin A was introduced into clinical practice in 1952 as a broad-spectrum antibiotic, the impact of polyketides on drug discovery has been revolutionary, and now approximatively $20 \%$ of the top-selling small molecule drugs are polyketides. ${ }^{1}$ Indeed, polyketides are arguably the most important class of natural products to human health, having been extensively mined, modified and manipulated to maximise therapeutic activity. Nature constructs such molecules through iterative assembly of a small 
number of building blocks followed by a series of post-synthetic modifications, ultimately creating myriad natural products with high levels of diversity in both structure and function (Figure 1a). ${ }^{2-5}$ Iterative strategies have also been extensively employed in synthesis, particularly in the construction of polyketides, which bear common repeat motifs such as propionates, acetates and deoxypropionates. ${ }^{6-8}$ In the case of polyacetates, which have a repeating 1,3-polyol motif, the iterative process has traditionally involved asymmetric allylation of an aldehyde, followed by alcohol protection and ozonolysis (Figure 1b). ${ }^{9,10}$ This process has been rendered particularly efficient through Krische's Ircatalysed enantioselective allylation of alcohols, although three steps per iteration are still required. ${ }^{1-}$ ${ }^{13}$ For example, in Garcia-Fortanet's synthesis of the reported structure of passifloricin A, ${ }^{9}$ four hydroxyl groups were introduced in 12 steps, and in Krische's synthesis of (+)-roxaticin, ${ }^{12}$ six hydroxyl groups were introduced but in just 9 steps by taking advantage of bidirectional chain synthesis. Acetate aldol reactions have also been developed, ${ }^{14}$ such as Carreira's silyl ketene acetal addition reaction (Figure 1b). ${ }^{15-17}$ Although highly enabling, the iterative aldol and allylation reaction sequences described above require three steps per hydroxyl group, suggesting that general approaches that provide more expediated routes to these motifs would have broad impact.

In recent years, we have developed iterative synthetic strategies that provide access to acyclic molecules containing multiple stereogenic centres through reagent-controlled homologation of boronic esters with lithium carbenoids, a process termed lithiation-borylation (Figure 1c).${ }^{18-24}$ Because the product of each homologation is itself a boronic ester, each iteration involves a single step, with no further protections or functional-group manipulations being required. This process utilized a range of carbenoid reagents (lithiated carbamates and benzoates) bearing different functionality and stereochemical configuration, thus enabling carbon chains to be 'grown' one atom at a time without purification of intermediates and with full stereocontrol, in a manner akin to a molecular assembly line. 1,2-Bis(boronic esters), derived from the enantioselective diboration of terminal alkenes, ${ }^{25}$ can also be engaged in lithiation-borylation reactions and they react selectively with lithiated carbamates and benzoates at the chain terminus to give 1,3-diols after boronic ester oxidation. ${ }^{26}$ We reasoned that conducting the homologation step with an enantioenriched carbenoid containing a butenyl unit would enable an iterative protocol consisting of 
alternating phases of diboration and homologation, where the alkene introduced in the homologation phase would be primed to undergo a subsequent asymmetric diboration (Figure 1d). The configuration of the stereogenic centres introduced during both lithiation-borylation and catalytic diboration would be independently controlled, making the process highly flexible and enabling stereochemical information to be essentially dialled-in, thus providing extended 1,3-polyols of any stereochemical permutation with equal efficiency. A further attractive feature of this strategy is that alcohol protection/deprotection steps, which are otherwise invariably employed in iterative syntheses of polyacetates, would be avoided. Thus, our proposed approach would only require one step per alcohol group, in contrast to prior approaches, ${ }^{9,12}$ which required three steps. Herein, we describe the success of this expedited approach to the 1,3-polyol motif, culminating in the first total synthesis of bahamaolide A in just 14 steps with full stereocontrol. Furthermore, we demonstrate that the utility of polyboronic ester intermediates is not limited to conversion into polyols, but extends to a much broader array of functionality, owing to well-developed methodology that allows the stereospecific transformation of boronic esters into diverse functional groups, in a manner akin to post-synthetic modifications in polyketide biosynthesis. 

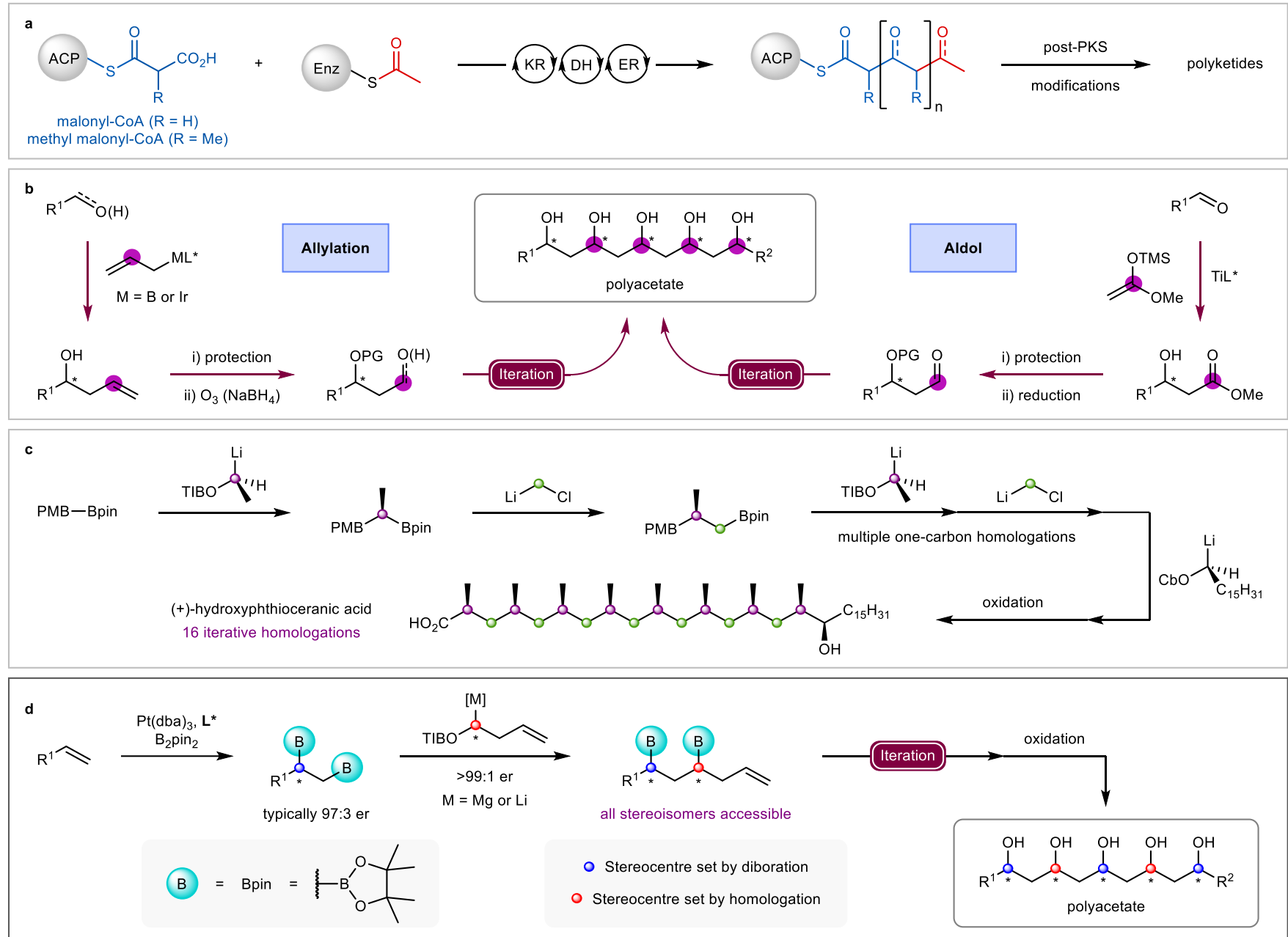

Figure 1 | Iterative approaches to the stereocontrolled synthesis of polyacetates. a Polyketide motifs are biosynthesised through the stepwise condensation of the growing chain with either malonyl-CoA or methylmalonyl-CoA. KR: ketoreductase, DH: dehydratase, ER: enoyl reductase, Enz: enzyme, ACP: acyl carrier protein, PKS: polyketide synthase. b Allylation: Asymmetric allylation of an aldehyde followed by alcohol protection and ozonolysis of the terminal alkene gives a $\beta$-alkoxyaldehyde which can be subjected to further iterative asymmetric allylation reactions. Krische's Ir-catalysed transfer hydrogenation reaction affords homoallylic alcohols in high er directly from the alcohol; protection and then ozonolysis with a reductive workup reveals the new terminal alcohol for the next iteration. Aldol: Enantioselective acetate aldol reaction followed by alcohol protection and reduction of the ester to an aldehyde can be applied iteratively in the synthesis of 1,3polyols. PG: protecting group. c Assembly-line synthesis: the iterative one-carbon homologation of boronic esters using reagent control for the synthesis of polydeoxypropionates has been applied to the total synthesis of several polyketide natural products. PMB: para-methoxylbenzyl, TIB: 2,4,6-triisopropylbenzoyl, $\mathrm{Cb}$ : diisopropylcarbamoyl. d This work: Iterative catalyst-controlled alkene diboration and reagent-controlled homologation of a boronic ester with a butenyl unit to construct 1,3-poly(boronic esters) with high stereocontrol. L*: 3,5-diisopropylphenyltaddol-PPh. 


\section{Results and discussion}

To test the feasibility of our proposed iterative diboration-homologation concept, we began with the Morken platinum-catalysed asymmetric diboration ${ }^{25}$ of a simple model substrate, 4-(but-3-en-1-yl)1,1'-biphenyl. This transformation gave bis(boronic ester) $\mathbf{1}$ in 77\% yield and 97:3 er, which could be enriched to $>99: 1$ er by recrystallization (Figure 2). For the homologation of the primary boronic ester of bis(boronic ester) $\mathbf{1}$, we required an enantioenriched butenyl carbenoid, and so we considered the application of $\alpha$-sulfinyl butenyl benzoate $\mathbf{2}$, from which the lithium or magnesium carbenoid could be generated by treatment with ${ }^{t} \mathrm{BuLi}$ or ${ }^{i} \mathrm{PrMgCl} \cdot \mathrm{LiCl}$, respectively. ${ }^{27}$ Both methods were tested, and it was found that the use of the magnesium carbenoid gave a superior yield when compared to that of the more reactive lithium carbenoid, presumably owing to the more promiscuous lithium carbenoid reacting with both the primary and the internal secondary boronic esters. Homologation of bis(boronic ester) 1 with either $\alpha$-sulfinyl benzoate $\mathbf{2}$ or $\mathbf{3}$ generated homoallylic boronic ester diastereomers $\mathbf{4}$ and $\mathbf{5}$, respectively, in comparable yields and with complete control of diastereoselectivity. In continuation of the iterative process and returning to the reaction step that initiated this sequence, asymmetric diboration of diastereomer 4 gave the tetraboronic ester 6 in 86\% yield and $>95: 5 d r$. Subsequent homologation with benzoate $\mathbf{2}$ gave homoallylic boronic ester $\mathbf{1 0}$ with high chemoselectivity for reaction at the terminal primary boronic ester over the three secondary boronic esters. The use of the enantiomeric ligands in the diboration and the diastereomeric $\alpha$-sulfinyl benzoates 2 or 3 in the homologation enabled all eight diastereoisomers 10-17 to be formed with equal ease, showing that there were no matched/mismatched effects in operation and that the individual processes showed full reagent/catalyst control. 


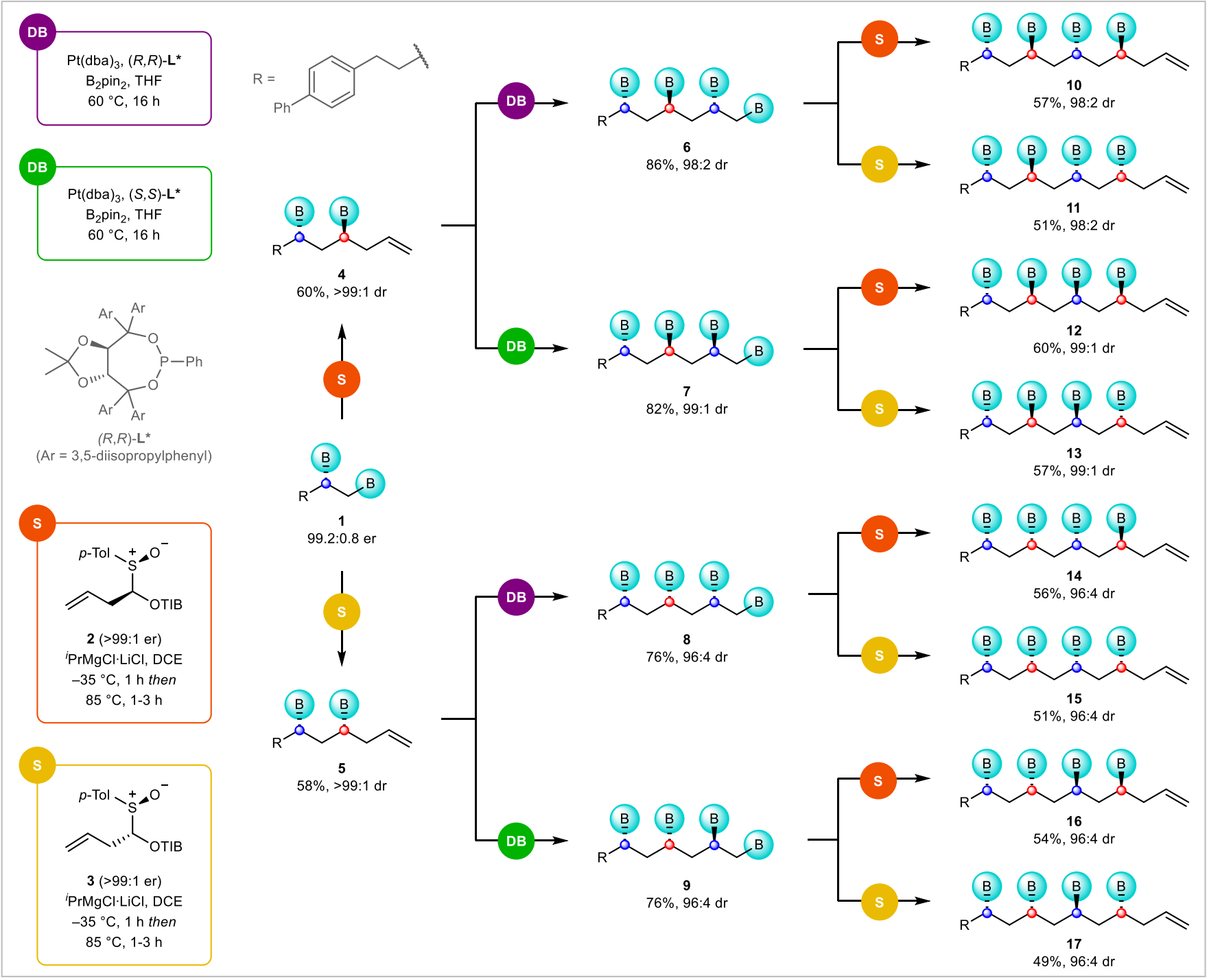

Figure 2 | Iterative synthesis of all eight diastereomers of a tetraboronic ester. The iteration involves (i) asymmetric diboration followed by (ii) reagent-controlled homologation of a boronic ester with a butenyl unit to construct 1,3-poly(boronic esters) with high stereocontrol. All quoted yields are of isolated material. Enantiomeric ratio for 1,2-bis(boronic ester) 1 and diastereomeric ratios for compounds 4-9 were measured by chiral HPLC analysis of the corresponding diols and tetraols, respectively. TIB: 2,4,6-triisopropylbenzoyl, DCE: 1,2dichloroethane, $p$-Tol: para-tolyl. 
Having established the feasibility of this iterative process, we aimed to apply it to the first total synthesis of bahamaolide A (18), a recently discovered member of the oxopolyene macrolide family bearing nine hydroxyl groups, which are 1,3-related..$^{28,29}$ Our retrosynthetic analysis led us to disconnect the polyene fragment to give the homoallylic silyl ether 19 (Figure 3). Within the 1,3-polyol portion, we recognized a $C_{2}$-symmetric octaol, and therefore considered homologation of a $C_{2}$-symmetric octaboronic ester $\mathbf{2 1}$ with different eastern and western building blocks $\mathbf{2 2}$ and $\mathbf{2 3}$. The octaboronic ester $\mathbf{2 1}$ could potentially be obtained in just 3 steps from 1,4-pentadiene (26) using our diboration-homologation iterative sequence in a bidirectional manner (Figure 1).

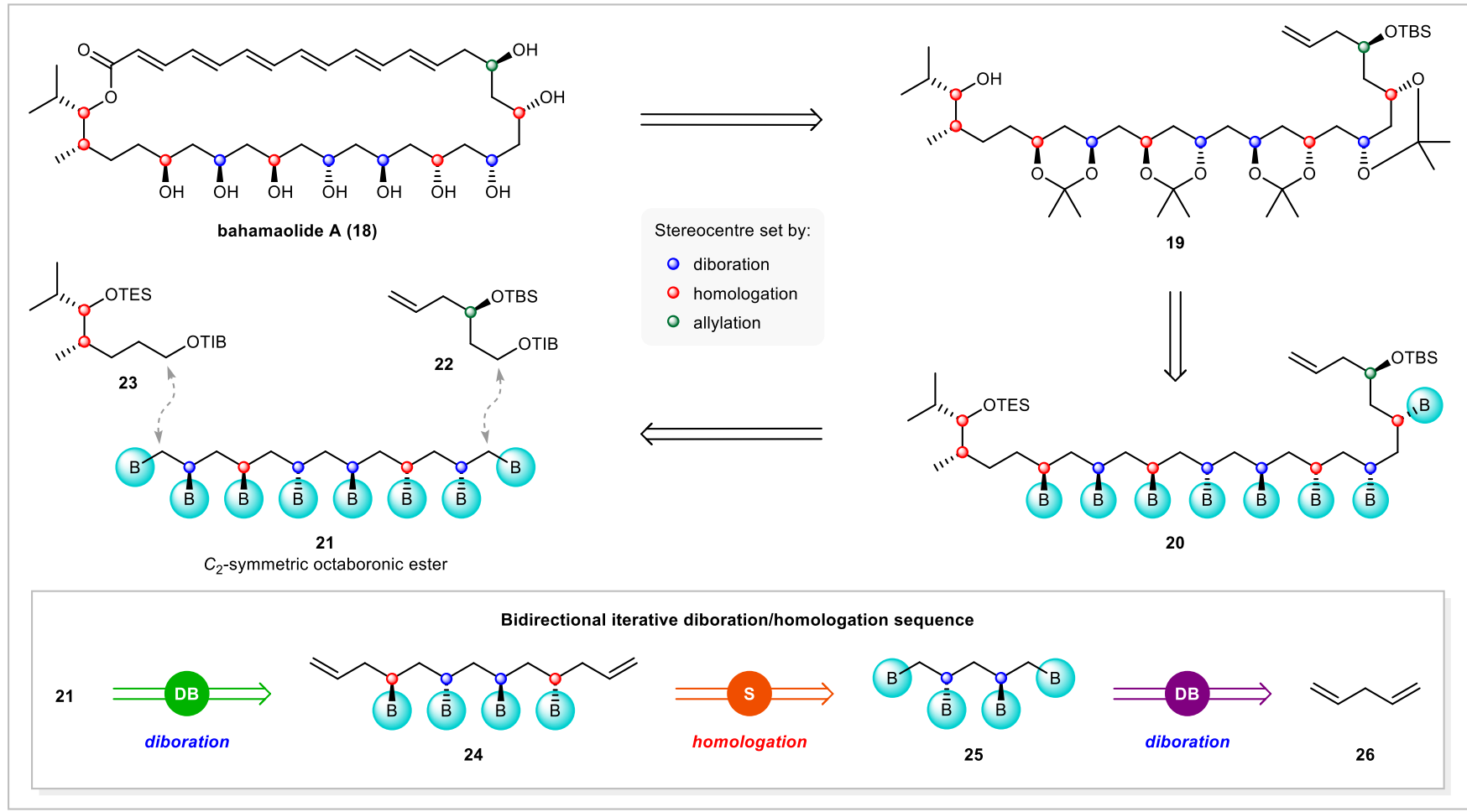

Figure 3 | Retrosynthetic analysis of bahamaolide A. Following disconnection of the polyene, recognition of a $\mathrm{C}_{2}$-symmetric polyol portion of bahamaolide A led us to consider an iterative bidirectional strategy. See $\mathrm{SI}$ for alternative strategies explored to install the polyene. 
Our synthetic campaign commenced with the double diboration of 1,4-pentadiene with Morken's asymmetric diboration protocol ${ }^{19}$ to form the $C_{2}$-symmetric $1,2,4,5$-tetraboronic ester $\mathbf{2 5}$, which was isolated in $89 \%$ yield and 95:5 $d r$ after chromatographic purification; the stereopurity was subsequently enhanced through recrystallisation to give 1,2,4,5-tetraboronic ester 25 as a single enantiomer and diastereomer in $60 \%$ yield (Figure 4a). X-ray analysis provided confirmation of the relative configuration of the tetraboronic ester. Using our homologation protocol, treatment of $\alpha$-sulfinyl benzoate 2 with ${ }^{i} \mathrm{PrMgCl} \cdot \mathrm{LiCl}$ followed by addition of the tetraboronic ester gave the desired doubly homoallylic tetraboronic ester $\mathbf{2 4}$ in $80 \%$ yield and with complete diastereoselectivity. Subsequent double diboration of doubly homoallylic tetraboronic ester $\mathbf{2 4}$ turned out to be more challenging: under the standard conditions, octaboronic ester $\mathbf{2 1}$ was obtained in $38 \%$ yield together with $27 \%$ of a heptaboronic ester side-product (see SI) derived from the diboration/hydroboration of tetraboronic ester 24. Increasing the concentration of the substrate from 0.1 to $1.0 \mathrm{M}$ greatly reduced the amount of the hydroboration side-product to just 7\%, enabling formation of octaboronic ester $\mathbf{2 1}$ in $64 \%$ yield. Thus, in just three steps, we were able to generate six of the stereocentres of bahamaolide and the functionalgroup handles required to append the east and west fragments. 


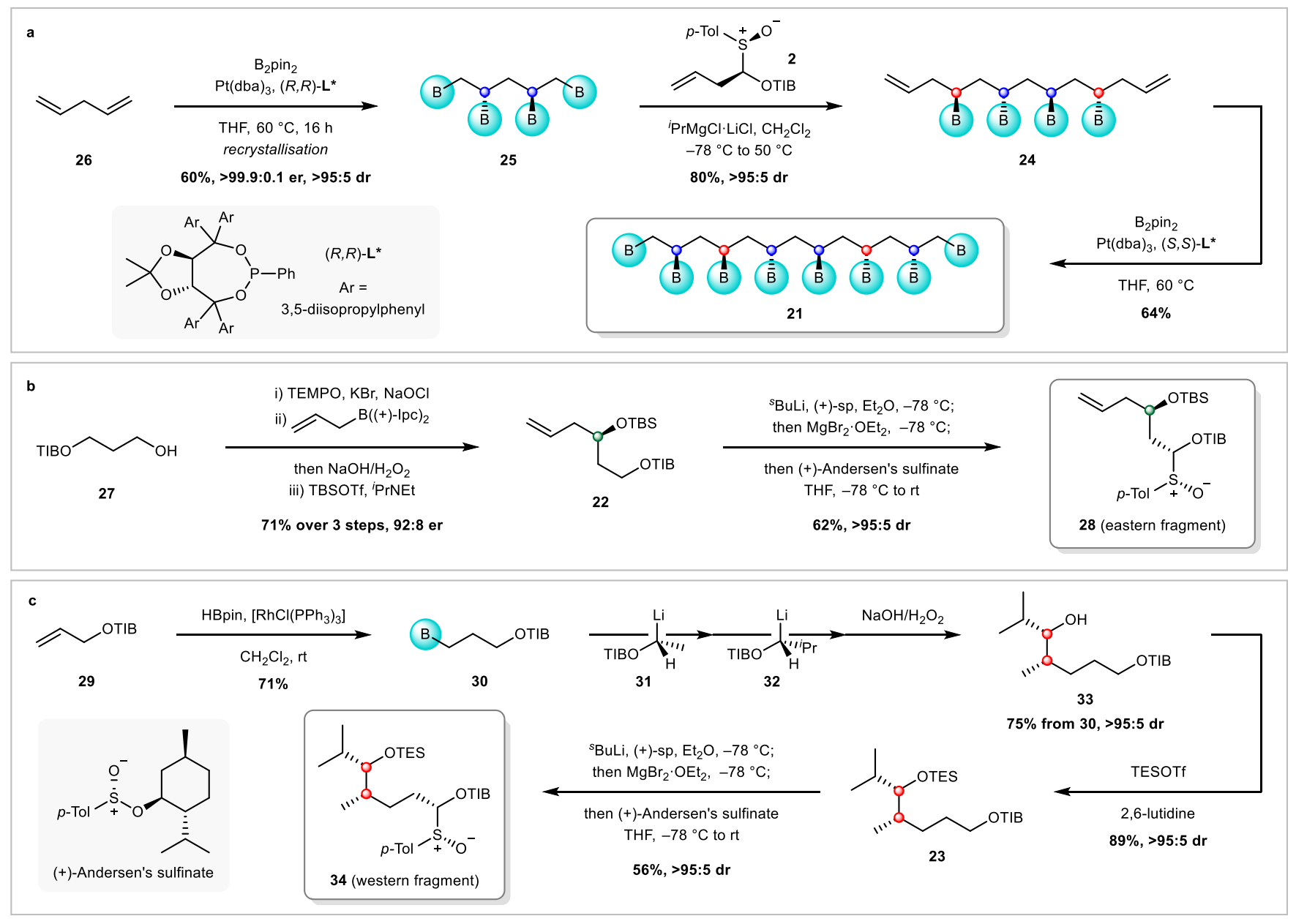

Figure 4 |a Synthesis of $C_{2}$-symmetric octaboronic ester 21. Iterative Morken diboration and homologation with $\alpha$-sulfinyl benzoate $\mathbf{2}$ bearing a butenyl unit in a bidirectional manner afforded octaboronic ester $\mathbf{2 1}$, setting six stereocentres in just three operations. Preparation of enantiopure chiral carbenoid precursors for the homologation of octaboronic ester 21. b Synthesis of eastern fragment 28. c Synthesis of western fragment 34. (+)-sp: (+)-sparteine.

Elaboration of octaboronic ester 21 required sequential homologation reactions with the eastern and western carbenoid precursors 28 and 34 (Figures $\mathbf{4 b}$ and $\mathbf{4 c}$ ). The eastern and western fragments both contain alcohol functionalities, but, as the western alcohol had to be deprotected selectively, ${ }^{12}$ it was protected as the TES ether 34, while the eastern alcohol was protected as the more stable TBS ether $\mathbf{2 8 .}$

The synthesis of eastern fragment 28 was achieved in three steps from alcohol 27 (Figure 4b). Oxidation followed by Brown asymmetric allylboration and protection gave benzoate $\mathbf{2 2}$ in $71 \%$ yield and 92:8 er. Subsequent enantioselective lithiation and reaction with enantiopure (+)-Andersen's sulfinate gave $\alpha$-sulfinyl benzoate $\mathbf{2 8}$ in $62 \%$ yield and as a single diastereomer. The western fragment 34 was generated from allyl benzoate 29 in four steps (Figure 4c). Hydroboration of 29 gave boronic 
ester 30, which was sequentially homologated with enantioenriched lithium carbenoids $\mathbf{3 1}$ and $\mathbf{3 2}$ and then oxidised to give alcohol $\mathbf{3 3}$ as a single diastereomer. The conversion of boronic ester $\mathbf{3 0}$ into alcohol 33 was highly efficient, proceeding in one-pot without purification of boronic ester intermediates. Protection of alcohol $\mathbf{3 3}$ as the TES silyl ether and installation of the sulfinyl group afforded $\alpha$-sulfinyl benzoate $\mathbf{3 4}$, also as a single diastereomer.

With the required $\alpha$-sulfinyl benzoates in hand, we turned our attention to the desymmetrisation of octaboronic ester 21 with $\alpha$-sulfinyl benzoate 28 (eastern fragment) (Figure 5). Thus, treatment of octaboronic ester $\mathbf{2 1}$ with 2.0 equivalents of $\alpha$-sulfinyl benzoate $\mathbf{2 8}$ and 2.1 equivalents of ${ }^{i} \mathrm{PrMgCl} \cdot \mathrm{LiCl}$ at $-78{ }^{\circ} \mathrm{C}$ for $1 \mathrm{~h}$, and then at $50{ }^{\circ} \mathrm{C}$ for $3 \mathrm{~h}$ - to allow 1,2-metallate rearrangement to occur-provided the desired boronic ester $\mathbf{3 5}$ in $36 \%$ yield, together with over-homologation product $\mathbf{3 6}$ and starting material 21 in $18 \%$ and $45 \%$ yield, respectively. The observed product ratio was in accordance with the expected statistical 1:2:1 distribution of $\mathbf{2 1 / 3 5 / 3 6}$. Increasing the number of equivalents of $\alpha$-sulfinyl benzoate 28 to 2.5 resulted in $47 \%$ yield of desired homoallylic octaboronic ester $35,23 \%$ yield of overhomologation product 36 and $26 \%$ yield of starting material 21, which represents the maximum achievable yield of desired product $\mathbf{3 5}$ when using octaboronic ester $\mathbf{2 1}$ as the limiting reagent. Although acceptable, we wanted to reduce the stoichiometry of sulfoxide $\mathbf{2 8}$ to create a more efficient process. Pleasingly, when the metalation and borylation phases of the reaction were performed at $-20{ }^{\circ} \mathrm{C}$, only 1.3 equivalents of $\alpha$-sulfinyl benzoate $\mathbf{2 8}$ were required to achieve a similar statistical distribution of products, giving desired boronic ester 35 in $45 \%$ yield, along with double homologation product $\mathbf{3 6}$ and starting material 21 in $21 \%$ and $20 \%$ yield, respectively. Indeed, ReactIR studies showed that in contrast to lithiated carbenoids, magnesiated carbenoids only reacted with boronic esters at temperatures above $-40{ }^{\circ} \mathrm{C}$, leading us to discover that $-20{ }^{\circ} \mathrm{C}$ was the optimum temperature for the metalation and borylation phases of the reaction. Finally, homologation of desymmetrised octaboronic ester $\mathbf{3 5}$ with $\alpha-$ sulfinyl benzoate 34 (western fragment) proceeded smoothly at $-20{ }^{\circ} \mathrm{C}$, to give homologation product 20 in $79 \%$ yield (90\% brsm).

We next investigated the simultaneous oxidation of all eight carbon-boron bonds to generate the required 1,3-polyol. A brief analysis revealed that the urea- $\mathrm{H}_{2} \mathrm{O}_{2}$ complex was best suited to this role, 
and, fortuitously, the TES protecting group was labile under these conditions, thus affording complete conversion of octaboronic ester $\mathbf{2 0}$ into the desired nonol. The crude polyol was protected as the tetra(acetonide) 19 in 58\% yield over 2 steps. This oxidation and protection sequence gave a molecule with increased chemical shift dispersion of NMR signals, thus enabling the diastereomeric ratio of $\mathbf{2 0}$ to be measured accurately: tetra(acetonide) 19 was generated in $>95: 5 d r$, demonstrating that the iterative synthesis and subsequent desymmetrisation of octaboronic ester $\mathbf{2 1}$ had proceeded with excellent diastereocontrol.

With the key 1,3-polyol fragment (19) constructed, the remaining steps concerned installation of the hexaene and closing the macrocycle. Different strategies were tested (see SI), but ultimately we found that a cross metathesis of tetra(acetonide) 19 with crotonaldehyde followed by a Horner-WadsworthEmmons (HWE) reaction worked well. The use of the Hoveyda-Grubbs II catalyst in the presence of crotonaldehyde transformed tetra(acetonide) $\mathbf{1 9}$ into the desired $\alpha, \beta$-unsaturated aldehyde $\mathbf{3 7}$ in $86 \%$ yield. The hexaeneoate is reportedly light- and air sensitive ${ }^{10}$ and so subsequent transformations were conducted under low/red light conditions and with minimal exposure to air. The HWE reaction with phosphonate $\mathbf{3 8}^{30,31}$ gave hexaene $\mathbf{3 9}$ with high selectivity for the six $E$-configured alkenes. Subsequent ester hydrolysis followed by Yamaguchi macrolactonisation, ${ }^{30}$ gave protected bahamaolide A. Finally, acid-catalysed global hydrolysis of the acetonides and TBS silyl ether completed our synthesis of bahamaolide A (18). Analytical data for the synthetic material $\left({ }^{1} \mathrm{H}\right.$ NMR, ${ }^{13} \mathrm{C}$ NMR, HRMS, optical rotation) matched those reported for the natural product. ${ }^{28}$ 


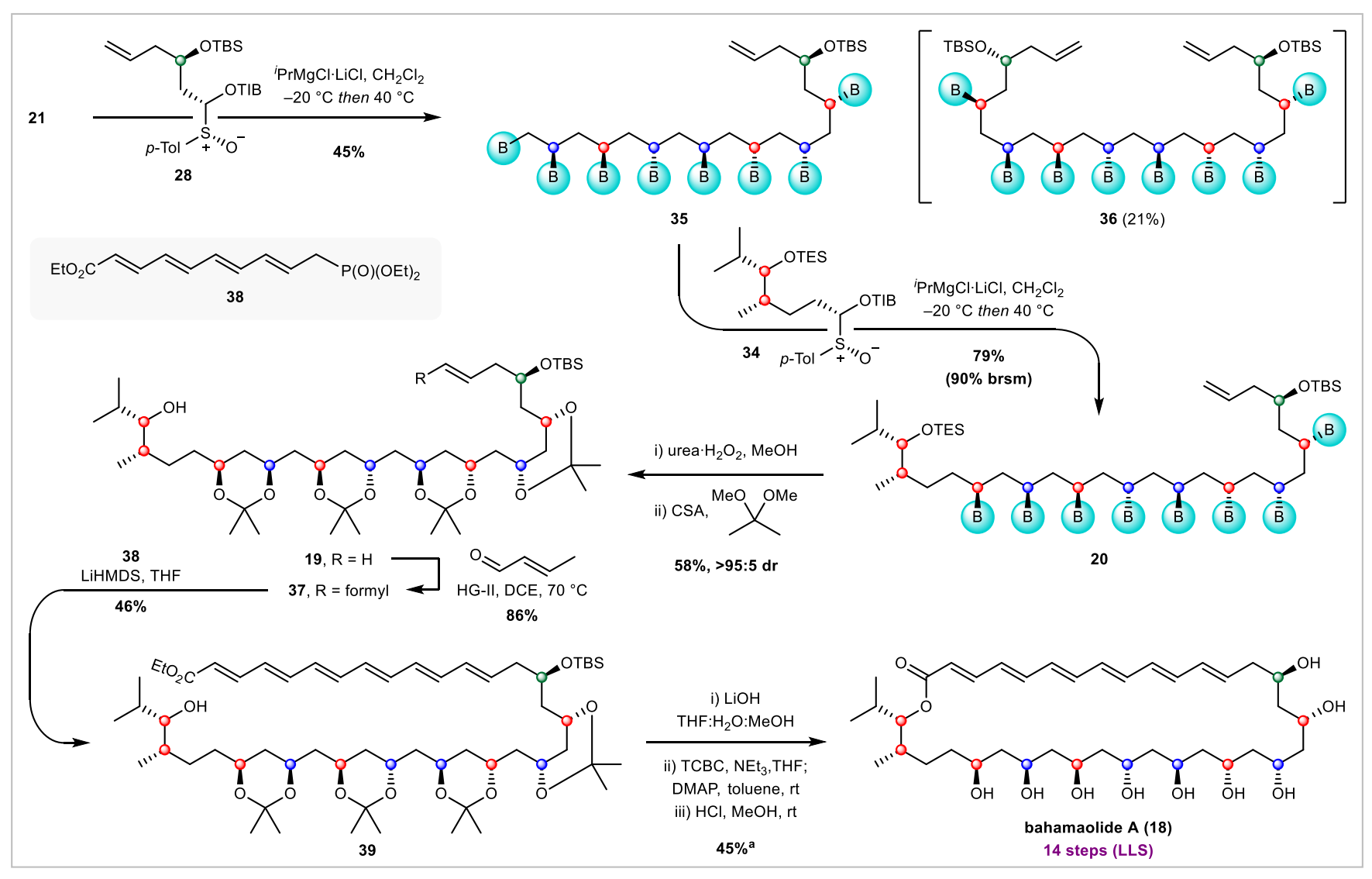

Figure 5 | Completion of the total synthesis of bahamaolide A from octaboronic ester 21, prepared in three steps from 1,4-pentadiene (see Figure 4). brsm: based on recovered starting material; CSA: camphorsulfonic acid; HG-II: Hoveyda-Grubbs $2^{\text {nd }}$ generation catalyst; DCE: 1,2-dichloroethane; TCBC: 2,4,6-trichlorobenzoyl chloride. ${ }^{a}$ yield determined by ${ }^{1} \mathrm{H}$ NMR analysis, using dimethoxymethane as internal standard.

The polyboronic esters we made en route to bahamaolide A offered additional opportunities in synthesis. Like the post-synthetic modifications that diversify polyketide chains following PKS assembly, ${ }^{32}$ the diverse selection of available stereospecific transformations of boronic esters allows us to prepare libraries of 1,3-functionalized molecules. ${ }^{33}$ Scaffolds presenting regularly spaced functional groups have applications in materials and medicine. ${ }^{34,35}$ For example, multivalent sialic acid-based constructs have been shown to bind to the surface of influenza virions, thus physically blocking their attachment to the cell-surface glycans of host cells and preventing infection. ${ }^{36,37}$ The nature of the scaffold presenting these sugar molecules, including flexibility and presentation pattern, has dramatic effects on the level of inhibition. ${ }^{38-40} \mathrm{We}$ therefore investigated a range of deborylative transformations to access libraries of 1,3-polyfunctionalised scaffolds.

The global functionalisation of polyboronic esters to give a homogeneous product requires access to 
highly efficient and robust methodology with high-fidelity transfer of stereochemical information, such as the urea- $\mathrm{H}_{2} \mathrm{O}_{2}$ system described above for stereospecific oxidation. Therefore, the polyboronic esters described herein also provide an ideal platform on which the test the efficiency and robustness of current deborylative functionalisation methodology. We therefore prepared a set of tetraboronic esters bearing different substituents and exhibiting different relative configurations of the carbon atoms bearing the boronic ester moieties by exploring the reactions of other carbenoid reagents with tetraboronic ester $\mathbf{2 5}$ (Figure 6a). Carbenoids derived from primary benzoates reacted with perfect diastereocontrol to provide secondary boronic esters $\mathbf{4 0 - 4 2}$ in high yields. The formation of tetraboronic ester $\mathbf{4 2}$ from $\boldsymbol{e n t}$ 25 again showed that no matched/mismatched effects occur during the homologation. Finally, we also tested lithiated secondary carbamates and benzoates, which gave the secondary-tertiary tetraboronic esters $\mathbf{4 3}$ and $\mathbf{4 4}$ in high yields and $d r$.

We then explored various transformations of the $C_{2}$-symmetric polyboronic ester 41 (Figure $6 \mathbf{b}$ ). Firstly, oxidation of tetraboronic ester 41 with urea- $\mathrm{H}_{2} \mathrm{O}_{2}$ complex gave the $C_{2}$-symmetric tetraol 45 in 93\% yield. Zweifel olefination ${ }^{41,42}$ initially proved problematic, as treatment of tetraboronic ester 41 with excess vinyllithium ( 2 equiv per boronic ester) at $-78{ }^{\circ} \mathrm{C}$ for 4 hours, followed by iodine and methanol gave a trivinyl product instead of the tetravinyl product. A brief exploration of reaction conditions revealed that temperature was the key issue and full boronate complex formation was only achieved upon warming to $0{ }^{\circ} \mathrm{C}$ for 30 minutes. Following addition of iodine, the tetravinyl product $\mathbf{4 6}$ was obtained in $93 \%$ yield and with complete stereocontrol. This experiment showed that in polyboronic esters, each boronic ester becomes progressively more hindered and less reactive as more of them are transformed into boronate complexes and full boronate complex formation is only achieved at elevated temperatures, even with highly reactive nucleophiles. The same Zweifel olefination could also be achieved with propenyl lithium, giving the tetrapropenyl product in $90 \%$ yield, which was subjected to global ozonolysis to form tetraketide $\mathbf{4 7}$ in $54 \%$ overall yield as a single stereoisomer. In addition, reaction of a lithiated enol carbamate with tetraboronic ester $\mathbf{4 1}$ under the same conditions gave the tetraenol carbamate in $52 \%$ yield, with subsequent elimination forming tetraalkyne $\mathbf{4 8}$ in $40 \%$ overall yield. ${ }^{43}$ Alkynes provide opportunities for further diversification of the 1,3-polyfunctionalised scaffold by reaction with azides in the CuAAC reaction; ${ }^{44,45}$ a reaction with a glycosylated azide gave 
polyglycoside 49 in $83 \%$ yield. Finally, the polyboronic ester could also be engaged in stereospecific polyarylations. This transformation was illustrated by treating tetraboronic ester $\mathbf{4 1}$ with lithiated furan and lithiated thiophene, transformations that gave the tetraaryl products $\mathbf{5 0}$ and $\mathbf{5 1}$, respectively. ${ }^{46}$ Attempts to carry out a global Matteson reaction failed, presumably because the chloromethyllithium reagent decomposed at the high temperatures required to achieve complete global boronate complex formation.

To probe the limit of this methodology, octaboronic ester $\mathbf{2 1}$ was further homologated in a chemoselective magnesiation-borylation reaction in 64\% yield and with $>95: 5 d r$ (Figure 6 c). Once again, the reaction showed perfect chemoselectivity for the two terminal primary boronic esters over the six secondary boronic esters. All eight boronic esters were transformed by using Zweifel olefination to give octaalkene $\mathbf{5 2}$ in $71 \%$ yield; the high yield was extraordinary considering the multiple steps that are involved in each transformation. Octaalkene $\mathbf{5 2}$ was subjected to olefin ring-closing metathesis using the Hoveyda-Grubbs II catalyst, giving tetracyclopentene $\mathbf{5 3}$ in $93 \%$ yield as a single diastereoisomer. Global olefin metathesis of polybutadiene has been reported previously, ${ }^{47}$ but because the relative configuration of stereocenters was not controlled, a stereorandom mixture of cyclopentenes would have been formed; our reaction gave a single $C_{2}$-symmetric diastereomer. 


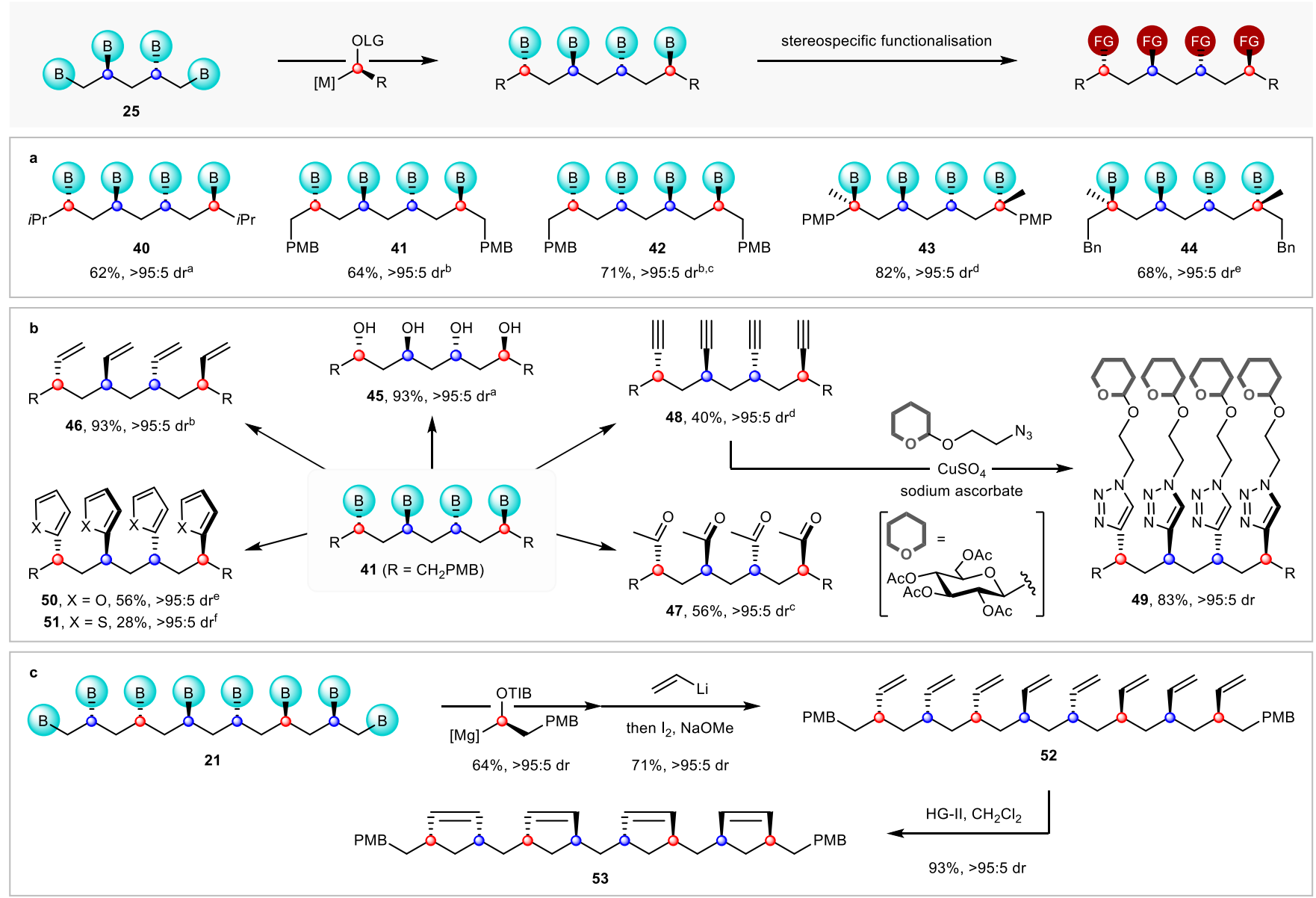

Figure 6 | Synthesis and functionalisation of polyboronic esters. a Synthesis of $C_{2}$-symmetric tetra(boronic esters) in two steps from 1,4-pentadiene. LG = 2,4,6-triisopropylbenzoyl (TIB) or diisopropylcarbamoyl (Cb). Conditions: ${ }^{a} \alpha$-sulfinyl benzoate, ${ }^{t} \mathrm{BuLi}, \mathrm{THF},-78{ }^{\circ} \mathrm{C}, 1 \mathrm{~h}, 65^{\circ} \mathrm{C}, 3 \mathrm{~h}$; ${ }^{\mathrm{b}} \alpha$-sulfinyl benzoate, ${ }^{i} \mathrm{PrMgCl} \cdot \mathrm{LiCl}, \mathrm{CH}_{2} \mathrm{Cl}_{2},-78$ ${ }^{\circ} \mathrm{C}, 1 \mathrm{~h}, 48{ }^{\circ} \mathrm{C}$, $3 \mathrm{~h} ;{ }^{\mathrm{c}}$ using tetraboronic ester ent-25, prepared using $(S, S)$-ligand; ${ }^{d}$ secondary benzylic carbamate, ${ }^{s} \mathrm{BuLi},{ }^{t} \mathrm{BuOMe},-78{ }^{\circ} \mathrm{C}, 2 \mathrm{~h}$, then add $25,-78{ }^{\circ} \mathrm{C}, 1 \mathrm{~h}, \mathrm{MgBr} 2 \cdot \mathrm{MeOH}$, warm to rt; ${ }^{\mathrm{e}} \alpha$-stannyl benzoate, ${ }^{n} \mathrm{BuLi}$, ${ }^{t} \mathrm{BuOMe},-78^{\circ} \mathrm{C}, 2 \mathrm{~h}$, then add $\mathbf{2 5},-78^{\circ} \mathrm{C}, 1 \mathrm{~h}$, warm to rt. PMB: para-methoxybenzyl; PMP: para-methoxyphenyl. b Stereospecific functionalisations of 1,3-tetraboronic esters. Simplified reaction conditions: ${ }^{\text {a }} \mathrm{urea}^{\mathrm{B}} \cdot \mathrm{H}_{2} \mathrm{O}_{2}$; ${ }^{\text {b }}$ vinyllithium, then $\mathrm{I}_{2}$, then $\mathrm{NaOMe}^{c}{ }^{c}$ prop-1-en-2-yllithium, then $\mathrm{I}_{2}$, then $\mathrm{NaOMe}$, then $\mathrm{O}_{3}$; ${ }^{d}$ vinyl diisopropylcarbamate, LDA, then $\mathrm{I}_{2}$, then ${ }^{t} \mathrm{BuLi}$, then $\mathrm{NH}_{4} \mathrm{Cl}$; ${ }^{e}$ furan-2-yllithium, then NBS; ${ }^{f}$ thiophen-2-yllithium, then NBS. c Homologation of octaboronic ester 21, global Zweifel olefination and global ring-closing metathesis. HG-II: Hoveyda-Grubbs $2^{\text {nd }}$ generation catalyst.

\section{Conclusions}

In conclusion, we have developed an iterative organoboron-based process for the synthesis of polyacetates, a common motif found in the polyketide class of natural products. The two-step iterative process, which can be deployed in a mono- or bidirectional manner, introduces masked 1,3-related diol motifs through stereospecific homologation of a boronic ester with a butenyl building block followed by stereoselective diboration of the terminal alkene. This synthetic strategy, which introduces a masked 1,3-diol unit in two steps, is more expeditious than current state-of-the-art methodology, which requires 
six steps (two iterations) to introduce the same fragment in the form of a protected 1,3-diol. Each stereogenic centre is independently controlled, either by the sulfoxide building block used in boronic ester homologation or the catalyst effecting diboration, and neither step suffers from matched/mismatched effects, thus enabling any stereoisomer to be obtained at will. The methodology has been applied to a 14-step synthesis of bahamaolide A with full stereocontrol, a route that includes the transformation of an octaboronic ester into the corresponding 1,3-polyol in one operation. The polyboronic esters can also be engaged in other stereospecific transformations, thus providing access to 1,3-related poly-alkenes, -alkynes, -ketones, and -aromatics with full stereocontrol, a level of versatility unmatched by other iterative strategies.

\section{REFERENCES}

1. Newman, D. J. \& Cragg, G. M. Natural Products as Sources of New Drugs from 1981 to 2014. J. Nat. Prod. 79, 629-661 (2016).

2. Staunton, J. \& Weissman, K. J. Polyketide biosynthesis: a millennium review. Nat. Prod. Rep. 18, 380-416 (2001).

3. Helfrich, E. J. N. \& Piel, J. Biosynthesis of polyketides by trans-AT polyketide synthases. Nat. Prod. Rep. 33, 231-316 (2016).

4. Hwang, S., Lee, N., Cho, S., Palsson, B. \& Cho, B.-K. Repurposing Modular Polyketide Synthases and Non-ribosomal Peptide Synthetases for Novel Chemical Biosynthesis. Front. Mol. Biosci. 87 (2020).

5. Ma, L. et al. Assembly Line and Post-PKS Modifications in the Biosynthesis of Marine Polyketide Natural Products. Compr. Nat. Prod. III 139-197 (2020).

6. Zheng, K., Xie, C. \& Hong, R. Bioinspired iterative synthesis of polyketides. Front. Chem. 3, $32(2015)$.

7. Lehmann, J. W., Blair, D. J. \& Burke, M. D. Towards the generalized iterative synthesis of small molecules. Nat. Rev. Chem. 201822 2, 1-20 (2018). 
8. ter Horst, B., Feringa, B. L. \& Minnaard, A. J. Iterative strategies for the synthesis of deoxypropionates. Chem. Commun. 46, 2535-2547 (2010).

9. García-Fortanet, J., Murga, J., Carda, M. \& Marco, J. A. On the structure of passifloricin A: Asymmetric synthesis of the $\delta$-lactones of (2Z,5S,7R,9S,11S)- and (2Z,5R,7R,9S,11S)tetrahydroxyhexacos-2-enoic acid. Org. Lett. 5, 1447-1449 (2003).

10. Zhang, Y., Arpin, C. C., Cullen, A. J., Mitton-Fry, M. J. \& Sammakia, T. Total Synthesis of Dermostatin A. J. Org. Chem. 76, 7641-7653 (2011).

11. Su Kim, I., Ngai, M.-Y. \& Krische, M. J. Enantioselective Iridium-Catalyzed Carbonyl Allylation from the Alcohol or Aldehyde Oxidation Level Using Allyl Acetate as an Allyl Metal Surrogate. J. Am. Chem. Soc. 130, 6340-6341 (2008).

12. Han, S. B., Hassan, A., Kim, I. S. \& Krische, M. J. Total Synthesis of (+)-Roxaticin via C-C Bond Forming Transfer Hydrogenation: A Departure from Stoichiometric Chiral Reagents, Auxiliaries, and Premetalated Nucleophiles in Polyketide Construction. J. Am. Chem. Soc. 132, 15559-15561 (2010).

13. Feng, J., Kasun, Z. A. \& Krische, M. J. Enantioselective Alcohol C-H Functionalization for Polyketide Construction: Unlocking Redox-Economy and Site-Selectivity for Ideal Chemical Synthesis. J. Am. Chem. Soc 138, 5467-5478 (2016).

14. Romea, P. \& Urpí, F. Stereoselective Acetate Aldol Reactions. in Modern Methods in Stereoselective Aldol Reactions (ed. Mahrwald, R.) 1-81 (Wiley, 2013).

15. Carreira, E. M., Singer, R. A. \& Lee, W. Catalytic, Enantioselective Aldol Additions with Methyl and Ethyl Acetate O-Silyl Enolates: A Chiral Tridentate Chelate as a Ligand for Titanium(IV). J. Am. Chem. Soc. 116, 8837-8838 (1994).

16. Kim, Y., Singer, R. A. \& Carreira, E. M. Total Synthesis of Macrolactin A with Versatile Catalytic, Enantioselective Dienolate Aldol Addition Reactions. Angew. Chemie Int. Ed. 37, 1261-1263 (1998). 
17. Rychnovsky, S. D., Khire, U. R. \& Yang, G. Total Synthesis of the Polyene Macrolide Roflamycoin. J. Am. Chem. Soc. 119, 2058-2059 (1997).

18. Leonori, D. \& Aggarwal, V. K. Lithiation-Borylation Methodology and Its Application in Synthesis. Acc. Chem. Res. 47, 3174-3183 (2014).

19. Burns, M. et al. Assembly-line synthesis of organic molecules with tailored shapes. Nature 513, 183 (2014).

20. Balieu, S. et al. Toward Ideality: The Synthesis of (+)-Kalkitoxin and (+)Hydroxyphthioceranic Acid by Assembly-Line Synthesis. J. Am. Chem. Soc. 137, 4398-4403 (2015).

21. Bootwicha, T., Feilner, J. M., Myers, E. L. \& Aggarwal, V. K. Iterative assembly line synthesis of polypropionates with full stereocontrol. Nat. Chem. 9, 896-902 (2017).

22. Wu, J. et al. Synergy of synthesis, computation and NMR reveals correct baulamycin structures. Nature 547, 436-440 (2017).

23. Pradeilles, J. A. et al. Odd-even alternations in helical propensity of a homologous series of hydrocarbons. Nat. Chem. 12, 475-480 (2020).

24. Yeung, K., Mykura, R. C. \& Aggarwal, V. K. Lithiation-borylation methodology in the total synthesis of natural products. Nat. Synth. 1, 117-126 (2022).

25. Coombs, J. R., Haeffner, F., Kliman, L. T. \& Morken, J. P. Scope and Mechanism of the PtCatalyzed Enantioselective Diboration of Monosubstituted Alkenes. J. Am. Chem. Soc. 135, $11222-11231(2013)$.

26. Fawcett, A. et al. Regio- and Stereoselective Homologation of 1,2-Bis(Boronic Esters): Stereocontrolled Synthesis of 1,3-Diols and Sch 725674. Angew. Chemie Int. Ed. 55, 1466314667 (2016).

27. Casoni, G. et al. $\alpha$-Sulfinyl Benzoates as Precursors to Li and Mg Carbenoids for the 
Stereoselective Iterative Homologation of Boronic Esters. J. Am. Chem. Soc. 139, $11877-$ 11886 (2017).

28. Kim, D. G. et al. Bahamaolides A and B, antifungal polyene polyol macrolides from the marine actinomycete streptomyces sp. J. Nat. Prod. 75, 959-967 (2012).

29. Lee, S.-H. et al. Bahamaolide A from the marine-derived Streptomyces sp. CNQ343 inhibits isocitrate lyase in Candida albicans. Bioorg. Med. Chem. Lett. 24, 4291-4293 (2014).

30. Evans, D. A. \& Connell, B. T. Synthesis of the Antifungal Macrolide Antibiotic (+)-Roxaticin. J. Am. Chem. Soc. 125, 10899-10905 (2003).

31. Mori, Y., Asai, M., Kawade, J. \& Furukawa, H. Total Synthesis of the Polyene Macrolide Antibiotic Roxaticin. II. Total Synthesis of Roxaticin. Tetrahedron 51, 5315-5330 (1995).

32. Hertweck, C. The Biosynthetic Logic of Polyketide Diversity. Angew. Chemie Int. Ed. 48, 4688-4716 (2009).

33. Sandford, C. \& Aggarwal, V. K. Stereospecific functionalizations and transformations of secondary and tertiary boronic esters. Chem. Commun. 53, 5481-5494 (2017).

34. Renaudet, O. \& Roy, R. Multivalent scaffolds in glycoscience: an overview. Chem. Soc. Rev. 42, 4515-4517 (2013).

35. Guan, X., Chen, F., Fang, Q. \& Qiu, S. Design and applications of three dimensional covalent organic frameworks. Chem. Soc. Rev. 49, 1357-1384 (2020).

36. Stadtmueller, M. N. et al. Evaluation of Multivalent Sialylated Polyglycerols for Resistance Induction in and Broad Antiviral Activity against Influenza A Viruses. J. Med. Chem. 64, $12774-12789$ (2021).

37. Bhatia, S., Dimde, M. \& Haag, R. Multivalent glycoconjugates as vaccines and potential drug candidates. Medchemcomm 5, 862-878 (2014).

38. Mammen, M., Choi, S.-K. \& Whitesides, G. M. Polyvalent Interactions in Biological Systems: 
Implications for Design and Use of Multivalent Ligands and Inhibitors. Angew. Chemie Int. Ed. 37, 2754-2794 (1998).

39. Bhatia, S. et al. Linear polysialoside outperforms dendritic analogs for inhibition of influenza virus infection in vitro and in vivo. Biomaterials 138, 22-34 (2017).

40. Vonnemann, J. et al. Size Dependence of Steric Shielding and Multivalency Effects for Globular Binding Inhibitors. J. Am. Chem. Soc. 137, 2572-2579 (2015).

41. Zweifel, G., Arzoumanian, H. \& Whitney, C. C. A convenient stereoselective synthesis of substituted alkenes via hydroboration-iodination of alkynes. J. Am. Chem. Soc. 89, 3652-3653 (1967).

42. Armstrong, R. J. \& Aggarwal, V. K. 50 Years of Zweifel Olefination: A Transition-Metal-Free Coupling. Synthesis (Stuttg). 49, 3323-3336 (2017).

43. Wang, Y., Noble, A., Myers, L. \& Aggarwal, V. K. Enantiospecific Alkynylation of Alkylboronic Esters. Angew. Chemie Int. Ed. 55, 4270-4274 (2016).

44. Mukherjee, C., Mäkinen, K., Savolainen, J. \& Leino, R. Chemistry and Biology of Oligovalent $\beta-(1 \rightarrow 2)$-Linked Oligomannosides: New Insights into Carbohydrate-Based Adjuvants in Immunotherapy. Chem. - A Eur. J. 19, 7961-7974 (2013).

45. Geng, Z., Shin, J. J., Xi, Y. \& Hawker, C. J. Click chemistry strategies for the accelerated synthesis of functional macromolecules. J. Polym. Sci. 59, 963-1042 (2021).

46. Bonet, A., Odachowski, M., Leonori, D., Essafi, S. \& Aggarwal, V. K. Enantiospecific sp2sp3 coupling of secondary and tertiary boronic esters. Nat. Chem. 6, 584-589 (2014).

47. Coates, G. W. \& Grubbs, R. Quantitative Ring-Closing Metathesis of Polyolefins. J. Am. Chem. Soc. 118, 229-230 (1996).

\section{ACKNOWLEDGEMENTS}


We thank H2020 ERC (670668) for financial support. H.-H.L. and T.B thank the Marie SkłodowskaCurie Fellowship programme for support (Horizon 2020, no. 746045 \& 626828 respectively). We thank N. Pridmore and H. Sparkes for X-ray analysis, D. J. Blair for useful discussions and C. P. Butts for assistance with NMR analysis.

\section{ADDITIONAL INFORMATION}

Affiliations: School of Chemistry, University of Bristol, Cantock's Close, Bristol, BS8 1TS, UK

\section{AUTHOR CONTRIBUTIONS}

S.G.A., J.M.B. and H.-H.L. contributed equally to this work. S.G.A., J.M.B., H.-H.L, A.F. and T.B. conducted the experimental work and analysed the data. All authors contributed to the preparation of the manuscript.

\section{CORRESPONDING AUTHOR}

Correspondence to v.aggarwal@ bristol.ac.uk

\section{COMPETING INTERESTS STATEMENT}

The authors declare no competing interests.

\section{GRAPHICAL ABSTRACT}

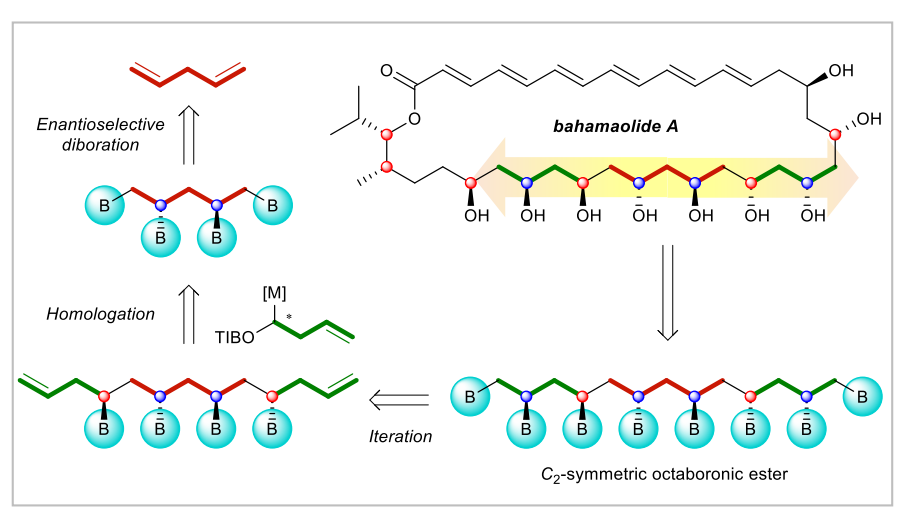

